\title{
Aggressive Variant of Synchronous Carcinoma Cervix and Ovary
}

\author{
Satyajeet Rath ${ }^{1}$, Rahat $\operatorname{Hadi}^{1}$, Ashish Singhal ${ }^{2}$, Mohammad Ali ${ }^{1}$ \\ Departments of ${ }^{1}$ Radiation Oncology and ${ }^{2}$ Surgical Oncology, Dr. Ram Manohar Lohia Institute of Medical Sciences, \\ Lucknow-226010, Uttar Pradesh, India.
}

\section{Corresponding Author: \\ Dr. Satyajeet Rath \\ Email: satyajeetrath@gmail.com}

This is an Open Access article distributed under the terms of the Creative Commons Attribution License (creativecommons.org/ licenses/by/3.0).

Received

Accepted

Published
January 31,2017

March 21, 2017

April 20, 2017

\begin{abstract}
Background: Synchronous malignancies of ovary and cervix are rare and generally entail poor prognosis. The optimal management is not well defined and varies from case to case basis. Case Report: We present a case of synchronous malignancy of cervix and bilateral ovary, which initially masqueraded as a case of malignant ascites pointing towards ovarian carcinoma, but after surgical (total abdominal hysterectomy and bilateral salpingooophorectomy along with omentectomy) and histopathological evaluation was found to be a case of dual malignancy. Patient also received neoadjuvant and adjuvant paclitaxel and carboplatin, followed by full dose whole pelvis radiotherapy. But, the disease was aggressive in nature and progressed rapidly to involve liver, lung and multiple bony sites, inspite of adequate treatment. Conclusion: The possibility of a synchronous malignancy should also be considered while working on genital malignancy.
\end{abstract}

Keywords: Carboplatin, Cervix Uteri, Hysterectomy, Ovarian Neoplasms, Pelvis.

\section{Introduction}

Concurrently occurring genital tumors of the cervix and ovary account for $0.025 \%$ of all genital tumours in females [1]. Ovarian and endometrial neoplasms occurring concurrently account for almost $40 \%$ of all synchronous malignancies occurring in genital tract, which carries a favourable prognosis in terms of early diagnosis and management [2]. Cases of genital malignancies involving three or four organs are very rare. We present here a case of aggressive variety of synchronously occurring cervical and ovarian carcinoma, with rapid spread of distant metastasis affecting liver, lung and bone.

\section{Case Report}

A50years gravida 1 para 1, presented with abdominal distension for 20 days and occasional complain of vaginal discharge. Ultrasound whole abdomen was suggestive of bilateral ovarian tumor with ascites. Contrast enhanced computed tomography (CECT) abdomen showed two well-defined septated cystic lesions seen in bilateral adnexal regions measuring $97 \times 92 \mathrm{~mm}$ and $52 \times 62 \mathrm{~mm}$ in right and left side respectively, with gross ascites and omental and mesenteric fat stranding and slightly bulky cervix [Fig.1,2]. Ascitic fluid was positive for malignant cells. Her baseline tumor markers were elevated; CA125: $273 \mathrm{U} / \mathrm{mL}$ (normal value: 0-35 units $/ \mathrm{mL}$ ), CA 19.9: 48.2 U/L (normal value: 0-37 units $/ \mathrm{mL}$ ) and CEA: $9 \mathrm{ng} / \mathrm{mL}$ (normal value: $<3 \mathrm{ng} / \mathrm{mL}$ ). Then she received four cycles of neoadjuvant paclitaxel and carboplatin. The disease load reduced both biochemically (CA 125 reduced to $63 \mathrm{U} / \mathrm{mL}$ ) and radiologically (CECT abdomen showing reduction in size of bilateral adnexa, $49 \times 51 \mathrm{~mm}$ and $24 \times 27 \mathrm{~mm}$, respectively on right and left side). Total abdominal hysterectomy with bilateral salpingo-oophorectomy was performed. Histopathology showed well-differentiated adenocarcinoma cervix with involved lower uterine segment. Bilateral ovaries showed adenocarcinoma. Bilateral fallopian tubes and parametrium were clear. 


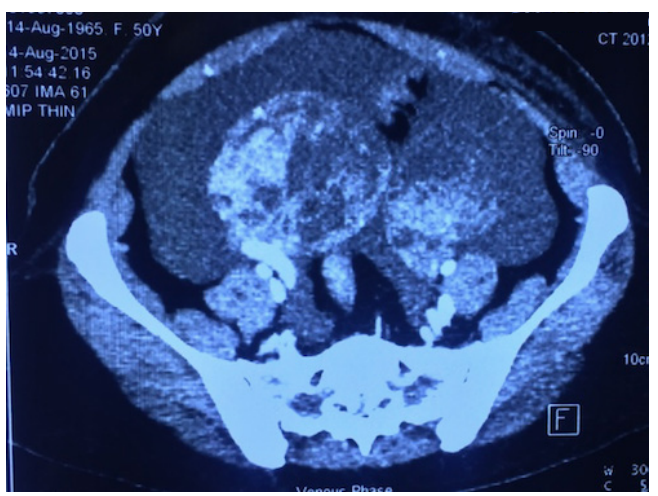

Fig.1: CECT abdomen showing bilateral ovarian adnexa.
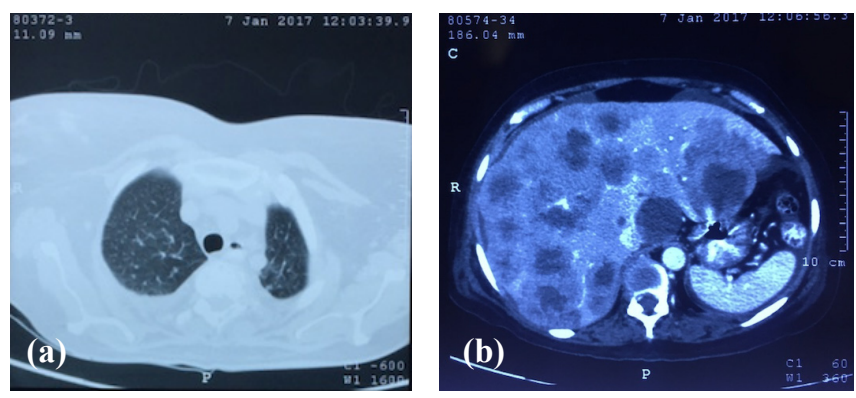

Fig.2(a): CECT thorax showing left lung upper lobe metastasis. (b): CECT abdomen axial cut showing multiple hypodense liver metastasis.

Two cycles of same adjuvant chemotherapy were given following which she was administered external beam radiotherapy 5000 cGy in 25 fractions followed by SORBO 800 cGy x 2 fractions. During three month follow up visit, she reported neck pain. MRI cervical spine showed wedging in C2, D2, D3, D5 and D11 with epidural soft tissue component at D2-3 suggestive of metastasis with marrow infiltration with spinal cord compression [Fig.3], which was given palliative radiotherapy 2000 cGy in five fractions. Patient reported back with symptoms of bilateral lower limb weakness (right $>$ left). Bone scan showed increased uptake in cervical vertebrae, sternum, right humerus, multiple thoracic vertebrae with liver metastasis [Fig.4]. CECT thorax and abdomen done subsequently showed multiple hepatic, pulmonary and bony metastases with multiple heterogeneously enhancing peri-portal, peri-pancreatic, and aortocaval nodes. Per speculum and per vaginum
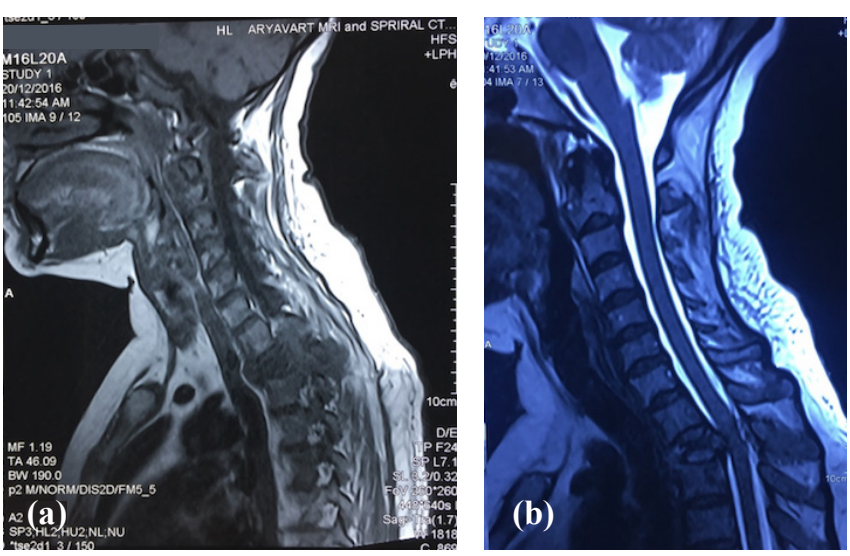

Fig.3(a): MRI T1 showing thoracic spine metastasis. (b): MRI T2 showing D2 vertebral collapse.

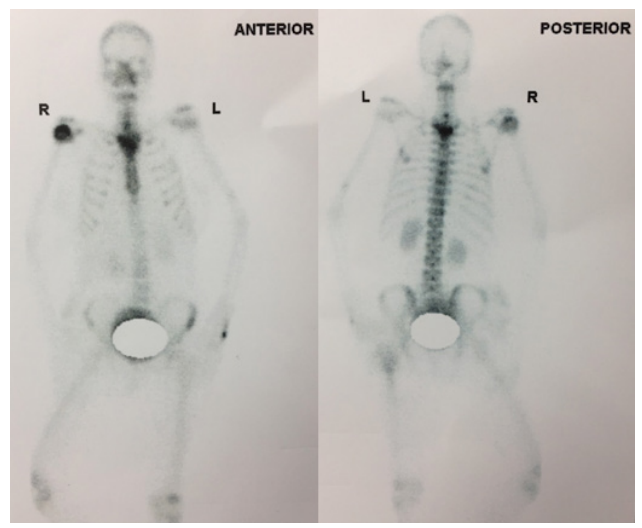

Fig.4: Bone scan showing multiple metastasis.

examination was normal. Follow up tumor markers were CA 125: 324 U/mL, CA 19.9: 521.4 U/L, CEA: $275 \mathrm{ng} / \mathrm{mL}$, AFP: $0.10 \mathrm{IU} / \mathrm{mL}$ and $\beta$-HCG: $0.5 \mathrm{IU} /$ $\mathrm{mL}$. Chemotherapy (paclitaxel and carboplatin) was started again in view of aggressive disease and adequate supportive treatment was given for palliating symptoms arising as a consequence of distant metastatic disease.

\section{Discussion}

Synchronous malignancies of the genital tract are rarely seen, most commonly involving ovary and endometrium [2,3], related with incessant ovulation, consequent oestrogen production, and continuous stimulation of the endometrium, with the consequent formation of neoplasms in 
these sites [4]. There are very few case reports in the literature reporting involvement of three (ovary, endometrium and cervix) or four (ovary, endometrium, cervix and fallopian tube) genital tract organs $[5,6]$. As such, cervix and ovarian malignancies are very rarely reported [7]. Although the present case is an incidental finding, the aggressive nature of the disease and the pathogenesis of the neoplastic process affecting the tissues with different embryological origins needs further evaluation. It is also important to distinguish multiple primary malignancies from metastatic disease because of the fact that overall survival and treatment would vary considerably [5]. Patients with multiple primary cancers have a high frequency of microsatellite instability (MSI), which might be a factor in the pathogenesis of this kind of disease [8]. The extensive use of markers like Ki$67, \mathrm{p} 16$ and p53 for cervical cancer, HNF 1- $\beta$, WT1, ER, PR p53, ARID1A, CK7, CK20, CK17 and PAX8 for ovarian cancer and p53, PTEN, $\beta$-catenin, PMS2 and MSH6 for endometrial carcinomas can further help in reaching a conclusive diagnosis [9]. Inspite of the continuous on-going research to identify genetic markers for development of synchronous tumors, studies have failed to address whether there is a common predisposing event. Authors have tried various modalities of treatment like surgery, radiotherapy and chemotherapy and their combination. Total pelvic exenteration has also been suggested for synchronous malignancies [10], but evidence is still scanty.

\section{Conclusion}

Synchronous malignancies are a rarity. Prompt diagnosis and well defined guidelines are needed to manage such cases. Combination of immunohistochemical, biomarkers and serum markers can overcome the current difficulties in screening and diagnosis of genital malignancies, particularly aggressive varieties and ensure the best possible management on case to case basis.

Contributors: SR: Manuscript writing, case management; RH: manuscript editing, case management; AS, MA: critical inputs into the manuscript and literature search. SR will act as guarantor. All authors approved the final version of the manuscript.

Funding: None; Competing interests: None stated.

\section{References}

1. Eisner RF, Nieberg RK, Berek JS. Synchronous primary neoplasms of the female reproductive tract. Gynecol Oncol. 1989;33:335-339.

2. Ayhan A, Yalçin OT, Tuncer ZS, Gürgan T, Küçükali T. Synchronous primary malignancies of the female genital tract. Eur J Obstet Gynecol Reprod Biol. 1992;45:63-66.

3. Zaino R, Whitney C, Brady MF, Degeest K, Burger RA, Buller RE. Simultaneously detected endometrial and ovarian carcinomas-a prospective clinico-pathologic study of 74 cases: a gynecologic oncology group study. Gynecol Oncol. 2001;83:355-362.

4. Tong SY, Lee YS, Park JS, Bae SN, Lee JM, Namkoong SE. Clinical analysis of synchronous primary neoplasms of the female reproductive tract. Eur J Obstet Gynecol Reprod Biol. 2008;136:78-82.

5. Pekin T, Yoruk P, Yildizhan R, Yildizhan B, Ramadan $\mathrm{S}$. Three synchronized neoplasms of the female genital tract: an extraordinary presentation. Arch Gynecol Obstet. 2007;276:541-545.

6. Saglam A, Bozdag G, Kuzey GM, Kuçukali T, Ayhan A. Four synchronous female genital malignancies: the ovary, cervix, endometrium and fallopian tube. Arch Gynecol Obstet. 2008;277:557-562.

7. Kambi DP, Mallikarjuna M, Santosh C, Abhishek V. Synchronous malignancies of ovary, fallopian tube and cervix: A rare case. International J Biomedical Advance Research. 2013;4:676-679.

8. Shannon C, Kirk J, Barnetson R, Evans J, Schnitzler M, Quinn $\mathrm{M}$, et al. Incidence of microsatellite instability in synchronous tumors of the ovary and endometrium. Clinical Cancer Research. 2003;9:1387-1139.

9. Kaspar HG, Crum CP. The utility of immunohistochemistry in the differential diagnosis of gynecologic disorders. Arch Pathol Lab Med. 2015;139:39-54.

10. Suciu N, Balescu I, Bacalbasa N. Total pelvic exenteration for synchronous cervical and ovarian tumour - a case report. Chirurgia (Bucur). 2015;110:486489. 